\title{
Efecto del Aumento de la Temperatura, Frecuencia de Alimentación y Ración de Alimento en el Crecimiento de Juveniles de Turbot Psetta maxima
}

\author{
Effect of Increased Temperature, Frequency and Feed Ration on Growth \\ of Juvenile Turbot Psetta maxima
}

${ }^{*, * *}$ Renzo Pepe-Victoriano; ${ }^{* * *}$ Alfonso Silva; ${ }^{* * * *, * * * * *}$ Alonso Vega; ${ }^{* * * * * * *, * * * * * * * *}$ Miguel Araya $\&{ }^{*, * * * * * * * *}$ Lorena Cornejo

PEPE-VICTORIANO, R.; SILVA, A.; VEGA, A.; ARAYA, M. \& CORNEJO, L. Efecto del aumento de la temperatura, frecuencia de alimentación y ración de alimento en el crecimiento de juveniles de turbot Psetta maxima. Int. J. Morphol., 30(3):902-907, 2012.

RESUMEN: Se determina la influencia del aumento de la temperatura $\left(17\right.$ a $\left.20^{\circ} \mathrm{C}\right)$, la ración $(2,5 \%$ a $3,5 \%$ del peso día $)$ y la frecuencia de alimentación (4 a 6 veces día), en el crecimiento y la supervivencia de juveniles de turbot (Psetta maxima) en un sistema productivo comercial durante 35 días. Los resultados muestran que el crecimiento de los juveniles provenientes del nuevo protocolo de cultivo experimental ensayado es mayor y muestra una tasa específica de crecimiento, significativamente diferente respecto a los cultivados con el protocolo base, sin afectar la supervivencia, la que se mantiene en $99,8 \%$. Se sugiere realizar mayores estudios para aislar o definir exactamente el efecto sinérgico de las variables definidas, así como complementar los resultados obtenidos con un estudio econométrico que evalúe su real aporte económico dentro de la cadena productiva del cultivo.

PALABRAS CLAVE: Producción de peces; Protocolo de cultivo; Supervivencia.

\section{INTRODUCCIÓN}

Los principales parámetros de producción en sistemas acuícola corresponden aquellos que regulan el crecimiento y la supervivencia de los individuos criados en cautiverio. Algunos son abióticos, o también llamados ambientales (Imsland et al., 2001; Tucker et al., 2006); mientras que otros son bióticos, o sea dependen de los atributos biológicos de la especie seleccionada para el cultivo (Lambert \& Dutil 2001; Morimoto et al., 2006). Así pues, si la combinación de estos parámetros es óptima entonces el crecimiento y la supervivencia de los individuos criados en cautiverio es maximizado y la producción garantizada.

Un parámetro ambiental determinante para el crecimiento del pez en cautiverio es la temperatura, la cual incrementa proporcional y positivamente la tasa de crecimiento hasta alcanzar su nivel suboptimo, después de los cual tiene efectos negativos (Corey et al., 1983; Jobling,
1993). Para juveniles de turbot el crecimiento máximo se obtiene a $22^{\circ} \mathrm{C}$ (Imsland et al., 2001; Van Ham et al., 2003), aunque este resultado podría variar dependiendo de la tasa de alimentación, tamaño y origen geográfico de los animales (Imsland et al., 2000).

Entre los parámetro bióticos, los factores relacionados con la nutrición resultan esenciales para el óptimo crecimiento y supervivencia de los peces criados en cautiverio (Pearson-Le Ruyet, 2010); entre ellos, la frecuencia y la ración de alimentación son considerados parámetros relevante para lograr una producción eficiente (Dwyer et al., 2002; Biswas et al., 2006; Tucker et al.).

La frecuencia de alimentación, depende del tamaño de los peces (Pearson-Le Ruyet). Aunque en teoría el aumento de la frecuencia de alimentación incrementa el creci-

\footnotetext{
Centro de Recursos Naturales y Medio Ambiente (CERENAYMA), Departamento de Biología, Universidad de Tarapacá, Arica, Chile.

** Programa de Doctorado en Ciencias, Mención Biología. Departamento de Biología, Universidad de Tarapacá, Arica, Chile.

*** Departamento de Acuicultura, Universidad Católica del Norte, Coquimbo, Chile.

**** Departamento Biología Marina, Universidad Católica del Norte, Coquimbo, Chile.

***** Programa de Doctorado en Biología y Ecología Aplicada, Universidad Católica del Norte, Coquimbo, Chile.

******* Programa de Doctorado en Ciencias Aplicadas Mención Sistemas Marinos Costeros, Universidad de Antofagasta, Antofagasta, Chile

******** Facultad de Recursos Naturales Renovables, Universidad Arturo Prat, Iquique, Chile.

********* Centro de Investigaciones del Hombre del Desierto (CIHDE), CONICYT-REGIONAL Arica, Chile.
} 
miento, existen límites biológicos y productivos, incluso en una misma cohorte o grupo etario (Folkvord \& Ottera, 1993). Por esta razón, durante el proceso de cultivo, la frecuencia de alimentación depende de la especie, de la edad y del estado fisiológico del pez (Jobling; Silva, 2010). Un exceso de comida o sobrealimentación disminuye la eficiencia alimenticia de los peces aumentando los costos productivos y la calidad del agua del cultivo (Lambert \& Dutil). Por otra parte, la ración de alimento o cantidad de alimento entregada en el día es importante para el crecimiento, pero ha sido poco estudiado. Puvanendran et al. (2003) muestra que la ración óptima para alimentar juveniles de lenguado Limanda ferruginea es 3 veces mayor que la descrita en su protocolo original de cultivo (1\%). Paralelamente la ración óptima de alimentos depende de parámetros abióticos, como la temperatura, y de otros relacionados con el protocolo de cultivo, como la frecuencia de alimentación (Van Ham et al.). Así pues conocer la frecuencia de alimentación y la ración de alimento óptima para los peces cultivados es importante, no sólo en términos de crecimiento de los peces, sino también por razones económicas y ambientales (Sumagaysay, 1998).

El turbot es el único pez marino de importancia económica cultivado industrialmente en Chile desde 1992 (Alvial \& Manríquez, 1999) y su producción se ha mantenido estable en un promedio anual de $305 \pm 41$ ton al año. Desde sus inicios el protocolo base original de cultivo ha estado en constante investigación en busca de una mejor eficiencia del sistema productivo acuícola (Person-Le Ruyet), con resultados experimentales variables, dependiendo de las condiciones y escala del cultivo. Así pues, para que los resultados de las investigaciones sean válidos y aplicables a escala comercial, las experimentaciones dirigidas a la optimización de técnicas de cultivo, debieran ser desarrolladas en lo posible al interior de la cadena productiva y en base a los volúmenes comerciales producidos en la empresa. En este contexto, este estudio no intenta desarrollar o adaptar un protocolo equivalente al que requiere un cultivo durante la fase de experimentación o piloto, como ocurre en otros peces planos (Silva), sino optimizar el protocolo o técnica base de cultivo intensivo utilizada por una empresa nacional, evaluando el efecto del incremento de la tempera- tura, frecuencia de alimentación y ración de alimentación en el crecimiento de juveniles de Psetta maxima cultivados intensivamente.

\section{MATERIAL Y MÉTODO}

Esta investigación fue ejecutada en una planta de cultivo nacional de turbot (SRC), ubicada en la zona de los Molles, zona central de Chile, utilizando el protocolo productivo base establecido por la empresa para la crianza de juveniles de turbot durante la temporada de invierno. Para evaluar el efecto del aumento de la temperatura, frecuencias de alimentación y ración de alimento sobre el crecimiento de turbot, desde 36 estanques en producción de $1,2 \mathrm{~m}^{3}$ cada uno en un ciclo de cultivo normal, se eligieron aleatoriamente 4 estanques, separándolos en 2 estanques controles con protocolo base normal ( $\mathrm{C} 1$ y $\mathrm{C} 2)$ y 2 experimentales con protocolo experimental modificado (E1 y E2) en los cuales se aumentaron la temperatura, ración y frecuencia de alimentación, en niveles previamente testeados en experiencias individuales previas con cada uno de los parámetros hechas en la misma empresa (Tabla I).

Así un total de 74.456 turbot (Psetta maxima) de un rango de peso inicial entre 0,78 a $1,92 \mathrm{~g}$ y 55 días de edad, se distribuyeron en 4 estanques de $1,2 \mathrm{~m}^{3}$, de fibra, color verde oscuro, $\left(1,6 \mathrm{~kg} \mathrm{~m}^{-3}\right)$ con flujo abierto de agua de mar (salinidad $34 \pm 0,5$ ups) e intercambio de 10 a $20 \mathrm{~L} \mathrm{~min}^{-1}$ al inicio y final de la experiencia y régimen de luz continua. Los peces fueron mantenidos a una temperatura normal de cultivo de $17,4{ }^{\circ} \mathrm{C}$ para luego, 5 días previos al inicio de la experiencia, subir paulatinamente la temperatura de los estanques experimentales (E1 y E2) a $20,5^{\circ} \mathrm{C}$ utilizando calefactores graduados al interior de los mismos. Durante los siguientes 35 días los peces fueron mantenidos a temperaturas promedios de $17,4 \pm 0,2$ y $20,5 \pm 0,3{ }^{\circ} \mathrm{C}$, alimentados a ración de $2,5 \%$ y $3,5 \%$ y con una frecuencia de 4 y 6 veces al día en los estanques control ( $\mathrm{C} 1$ y C2) y experimentales (E1 y E2) respectivamente. Durante la experiencia la concentración de oxígeno del agua de los estanques se

Tabla I. Estado inicial del cultivo de juveniles de P. maxima en estanques controles (Protocolo base) y experimentales para la determinación del efecto del aumento de la temperatura, frecuencia, y ración alimenticia.

\begin{tabular}{lcccccccc}
\hline Estanques & $\begin{array}{c}\text { Edad } \\
(\mathbf{d} \text { ías })\end{array}$ & n peces & $\begin{array}{c}\text { Biomasa } \\
(\mathbf{K g})\end{array}$ & $\begin{array}{c}\text { Peso } \\
(\mathbf{g})\end{array}$ & $\begin{array}{c}\text { DE Peso } \\
(\mathbf{g})\end{array}$ & $\begin{array}{c}\text { Frecuencia } \\
\text { Alimenticia }\end{array}$ & $\begin{array}{c}\text { Porción } \\
\text { Alimento }(\%)\end{array}$ & $\begin{array}{c}\text { Temperatura } \\
\left({ }^{\mathbf{0}} \mathbf{C}\right)\end{array}$ \\
\hline C1 & 55 & 2.401 & 2,040 & 0,85 & 0,09 & 4 veces al día & $2,5 \%$ & 17,4 \\
C2 & 55 & 2.301 & 1,863 & 0,81 & 0,09 & 4 veces al día & $2,5 \%$ & 17,4 \\
E1 & 55 & 2.301 & 1,863 & 0,81 & 0,08 & 6 veces al día & $3,5 \%$ & 20,5 \\
E2 & 55 & 2.323 & 1,928 & 0,83 & 0,11 & 6 veces al día & $3,5 \%$ & 20,5 \\
\hline
\end{tabular}


mantuvo en 5,4 $\mathrm{mg} \cdot \mathrm{L}^{-1}$ promedio, tanto para los estanques control como para los estanques experimentales.

Los peces fueron alimentados manual y diariamente con pellet confeccionado en la misma empresa y con un contenido de $53 \%$ proteína y $15 \%$ lípidos. Diariamente el alimento no consumido fue sifonado en cada estanque. Al inicio, cada cinco días y al final de la experiencia, se determinó el peso individual en gramos de 60 peces de cada uno de los estanques, al comienzo de la jornada laboral. Se calculó la tasa de crecimiento específico (SGR) en peso como porcentaje de cambio del peso cuerpo diario, de acuerdo a Ricker (1979): $\operatorname{SGR}(\%)=[[\ln w f-\ln w i] / t] * 100 ;$ Donde: $w i=$ Peso inicial, $\mathrm{wf}=$ Peso final y $\mathrm{t}=$ Tiempo en días. Igualmente se estimó el porcentaje de incremento en peso (Ricker) utilizando la ecua- ción: IP $(\%)=[[w f-w i] /$ wi $] * 100$. La supervivencia se determinó a través del análisis de las mortalidades diarias, por estanque, las cuales fueron contabilizadas al final de la experiencia, obteniendo el número real de juveniles vivos. Esta se expresa como porcentaje de supervivencia (Ricker), cuya expresión es: $\mathrm{S}(\%)=[\mathrm{nf}-\mathrm{ni}] * 100$, Donde: $\mathrm{ni}=$ Número de individuos inicial y $\mathrm{nf}=$ Número de individuos final.

Para comparar los tratamientos experimentales y control se utilizó el análisis de varianza (ANDEVA) de un factor y de medidas repetidas. La normalidad de los datos y la homocedasticidad de las varianzas fueron comprobadas a través de la prueba de Barlett y Lilliefors (Sokal \& Rohlf, 1981). Cuando el ANDEVA detectó diferencias significativas se aplicó la prueba a posteriori de Tukey (Sokal \& Rohlf).

Tabla II. Incremento en peso (IP\%) y supervivencia (S\%) de juveniles de turbot (P. maxima) cultivados con el protocolo base y experimental.

\begin{tabular}{lccccc}
\hline Protocolo & $\begin{array}{c}\text { Temperatura }^{\circ} \mathbf{C} / \\
\text { Frecuencia/Ración }\end{array}$ & $\begin{array}{c}\text { Peso Inicial } \\
(\mathbf{g}) \pm \mathbf{D E}\end{array}$ & $\begin{array}{c}\text { Peso Final } \\
(\mathbf{g}) \pm \mathbf{D E}\end{array}$ & IP (\%) & S (\%) \\
\hline Base $(\mathrm{C})$ & $17,4^{\circ} \mathrm{C} / 4 / 2,5 \%$ & $0,83 \pm 0,09$ & $6,00 \pm 0,34$ & 622,89 & 99,85 \\
Experimental (E) & $20,5^{\circ} \mathrm{C} / 6 / 3,5 \%$ & $0,82 \pm 0,09$ & $7,00 \pm 0,46$ & 753,65 & 99,83 \\
\hline
\end{tabular}

$\mathbf{A}$
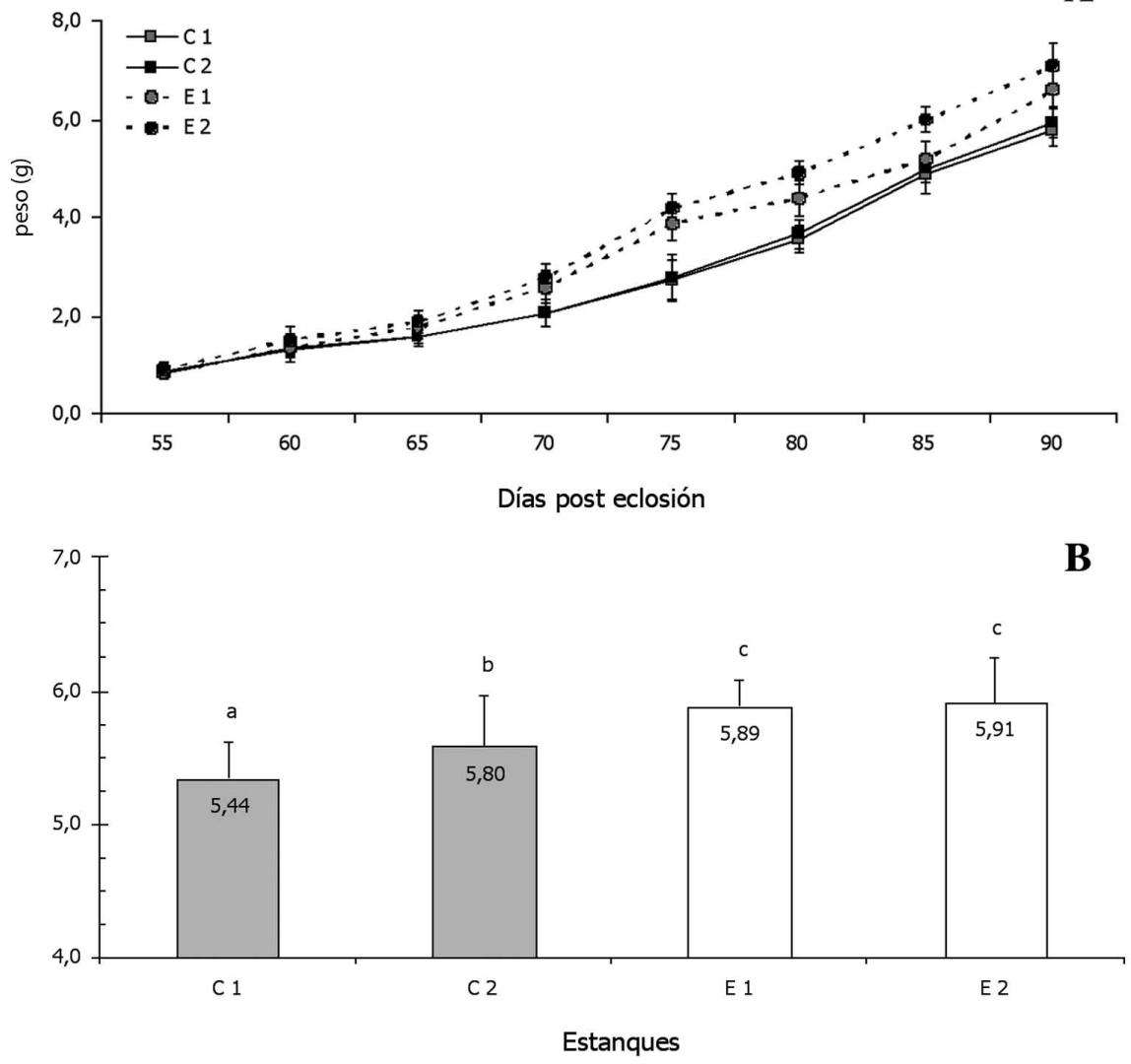

Fig. 1. Incremento en peso (g) (A) y tasa de crecimiento especifico en peso (SGR) (B) de juveniles de turbot (P. maxima) criados con el protocolo de cultivo base y experimental. 


\section{RESULTADOS}

Los resultados de la experiencia productiva muestran que el peso de los juveniles criados en ambos grupos aumentó durante todo el período de estudio (Fig. 1 y Tabla II). Con el protocolo base el aumento en peso final fue de $622,89 \%$ y con el protocolo experimental de $753,65 \%$. Estos últimos además, presentan un incremento en peso significativamente mayor que los criados con el protocolo base $($ ANDEVA F $(3,236)=69,57 ; \mathrm{P}<0,001)$, lo cual es más notorio desde el día 70 post eclosión (Fig. 1A). Al final del experimento, la tasa de crecimiento específico (SGR) de los peces criados en los estanques donde se aplicó el protocolo experimental fue significativamente mayor que la tasa de crecimiento de aquellos peces criados en los estanques donde se utilizó el protocolo base (ANOVA F $(3,236)=118,0 ; \mathrm{P}<$ 0,001) (Fig. 1B). La supervivencia final en ambos grupos fue alta $(98,8 \%)$ y no muestra diferencias significativas.

\section{DISCUSIÓN}

Estudios en diferentes especies indican que ante un aumento de la temperatura la SGR incrementa y en ocasiones covaría con el aumento de la ración y frecuencias alimenticias (Vam Ham et al.). De nuestra experiencia podemos concluir que el aumento de los factores mencionados produce un efecto acumulativo final significativo en el crecimiento en peso y la SGR en los juveniles de turbot cultivados comercialmente. Este efecto sinérgico ha sido observado también cuando se consideran distintos parámetros ambientales como moduladores del incremento en peso de los peces (Rombough, 1996), aunque como ya ha sido reportado, es el incremento en temperatura el que acelera el metabolismo del pez obteniendo este, una mayor posibilidad de ingesta alimenticia (Jobling; Van Ham et al.). De la experiencia no es posible colegir cual es el factor más relevante al respecto, aunque un estudio reciente realizado por los mismos autores en juveniles de turbot, demuestra que el crecimiento es influenciado principalmente por la temperatura y en menor medida por la frecuencia alimenticia y la ración de alimento (Pepe, 2010). Por otro lado estudios realizados en $P$. maxima y otros peces marinos (e.g., Mola mola), demostraron que la frecuencia de alimento es más importante que la ración de alimento para el crecimiento de juveniles (Wang et al., 1998; Saether \& Jobling, 1999). Así un aumento en la ración de alimento en juveniles de turbot de 70-100 g, no afectó la tasa de crecimiento de los peces, mientras que al aumentar la frecuencia de alimentación el crecimiento aumentó (Saether $\&$ Jobling). En este contexto, el manejo de la frecuencia y la ración requieren de estudios complementarios que evalúen los procesos fisiológicos (e.g., tiempo de digestión) y los mecanismos de captura del alimento, lo cual afinaría la exactitud de los indicadores de conversión del alimento disminuyendo los costos de producción (MeyerBurgdorff et al., 1989). Ello considerando que existe una variabilidad intrínseca del protocolo durante las etapas del proceso de cultivo de una especie o entre protocolos de distintas especies (Ruohonen et al., 1998), que podría determinar el rango óptimo de los parámetros ambientales y biológicos.

Respecto al rango de temperatura utilizado y considerando que en ambos experiencias el crecimiento fue positivo, podemos concluir que este se encuentra dentro del óptimo de temperaturas para el crecimiento de juveniles de turbot en Chile (Hasan \& MacIntoch, 1991).

En general, una forma de validar la modificación de un protocolo base además del incremento en peso y el crecimiento, es la supervivencia. Así, Pastor \& Grau (2002) señalan que la selección de una frecuencia y ración adecuada de alimento para juveniles de Dentex dentex suministrado a través de alimentadores automáticos, dependió de que se alcanzarán niveles de supervivencia por sobre el $92 \%$ final. En este contexto el protocolo experimental probado, no difirió significativamente del protocolo base que se desea optimizar en términos de supervivencia final, la que se mantuvo alta y cercana al 99\%, coincidiendo con otros estudios que han evaluado experimentalmente parámetros ambientales y nutricionales en P. maxima (Person-Le Ruyet).

Si bien es cierto una ganancia en peso promedio de 1 $\mathrm{g}$ del protocolo experimental en la etapa de hatchery podría traducirse en el ahorro final de 1 a 2 meses de operación en la llegada a talla de cosecha de turbot, también la implementación del protocolo experimental con lleva comparativamente mayores costos operativos (e.g., tiempo, dinero, energía), que deben ser debidamente evaluados caso a caso, para determinar su conveniencia económica. En conclusión, se prueba que el efecto del aumento de la temperatura, frecuencia de alimentación y ración de alimento del protocolo base utilizado para la producción comercial de juveniles de turbot (Psetta maxima) mejora significativamente el crecimiento sin afectar la supervivencia, en un sistema de cultivo intensivo. Sin embargo un cambio en el protocolo base debiera decidirse a la luz de los resultados arrojados por un estudio econométrico previo, que evalúe su real aporte económico dentro de la cadena productiva del cultivo. 
PEPE-VICTORIANO, R.; SILVA, A.; VEGA, A.; ARAYA, M. \& CORNEJO, L. Efecto del aumento de la temperatura, frecuencia de alimentación y ración de alimento en el crecimiento de juveniles de turbot Psetta maxima. Int. J. Morphol., 30(3):902-907, 2012.

PEPE-VICTORIANO, R.; SILVA, A.; VEGA, A.; ARAYA, M. \& CORNEJO, L. Effect of increased temperature, frequency and feed ration on growth of juvenile turbot Psetta maxima. Int. J. Morphol., 30(3):902-907, 2012.

SUMMARY: The influence of water temperature $\left(17\right.$ and $\left.20^{\circ} \mathrm{C}\right)$, frequency $(4$ and 6$)$ and feed ration level $(2.5 \%$ and $3.5 \%)$ on the growth and survival of juvenile turbot (Psetta maxima) in a commercial productive system was investigated over 35 days. The results indicate that fish from the experimental protocol exhibited better growth and significant difference in specific growth rate than those raised with basic protocol, without affecting survival that remains at $99.8 \%$. It is suggested further studies to isolate or define exactly the synergistic effect of the variables defined and complement the results obtained with an econometric study which evaluate their real economic contribution within the productive chain of culture.

KEY WORDS: Fish production; Culture protocol; Survival.

\section{REFERENCIAS BIBLIOGRÁFICAS}

Alvial, A. \& Manríquez, J. Diversification of flatfish culture in Chile. Aquaculture, 176(1):65-73, 1999.

Biswas, G.; Jena, J. K.; Singh, S. K.; Patmajhi, P. \& Muduli, H. K. Effect of feeding frequency on growth, survival and feed utilization in mrigal, Cirrhinus mrigala, and rohu, Labeo rohita, during nursery rearing. Aquaculture, 254(1-4):211-8, 2006.

Corey, P. D.; Leith, D. A. \& English, M. J. A growth model for coho salmon including effects of varying ration allotments and temperature. Aquaculture, 30(1-4):125-43, 1983.

Dwyer, K. S.; Brown, J. A.; Parrish, C. \& Lall, S. P. Feeding frequency affects food consumption, feeding pattern and growth of juvenile yellowtail flounder (Limanda ferruginea). Aquaculture, 213(1-4):279-92, 2002.

Folkvord, A. \& Ottera, H. Effects of initial size distribution, day length, and feeding frequency on growth, survival and cannibalism in juvenile Atlantic cod (Gadus morhua L.). Aquaculture, 114(3-4):243-60, 1993.

Hasan, M. R. \& MacIntoch, D. J. Effect of environmental temperature and feeding rate on the growth, food utilization and body composition of common carp (Cyprinus carpio L.) fry. In: Kaushik, S. W. \& Luquet, P. Fish Nutrition in Practice. Paris, Les Colloques, 1991. pp.767-78. V. 61.

Imsland, A.; Foss, A.; Naevdal, G.; Cross, T.; Bonga, S. W.; Ham, E. A. \& Stefansson, S. O. Countergradiet variation in growth and food convertion efficiency of juvenile turbot. J. Fish Biol., 57(5):1213-26, 2000.

Imsland, A. K.; Foss, A.; Gunnarsson, S.; Berntssen, M. H. G.; FitzGerald, R.; Bonga, S. W.; Ham, E v.; Naevdal, G. \& Stefansson, S. O. The interaction of temperature and salinity on growth and food conversion in juvenile turbot (Scophthalmus maximus). Aquaculture, 198(3):353-67, 2001.

Jobling, M. Bioenergetics: feed intake and energy partitioning. En: Ranking, J. C. \& Jensen, F. B. Fish ecophysiology. London, Chapman \& Hall, 1993. pp.1-44.
Lambert, Y. \& Dutil, J. Food intake and growth of adult Atlantic cod Gadus morhua L. reared under different conditions of stocking density, feeding frequency and size-grading. Aquaculture, 192(2-4):233-47, 2001.

Meyer-Burgdorff, K. H.; Osman, M. F. \& Gunther, K. D. Energy metabolism in Oreochromis niloticus. Aquaculture, 79(14):283-91, 1989.

Morimoto, P.; Hosokawa, H. \& Masumoto, T. Effects of dietary supplementation with feeding stimulants on yellowtail Seriola quinqueradiata (Temminck \& Schlegel; Carangidae) protein digestion at low water temperatures. Aquaculture Res., 37(4):366-73, 2006.

Pastor, E. \& Grau, A. Efecto de la frecuencia de alimentación en alevines de dentón (Dentex dentex Linnaeus, 1758) durante la fase de preengorde. Bol. Inst. Esp. Oceanogr., 18(1-4):115-7, 2002.

Pepe, R. Influencia del aumento de la temperatura, frecuencia de alimentación y ración de alimento, en la optimización de la técnica base de cultivo de juveniles de turbot (Psetta maxima Linneaus, 1758). Tesis de Magíster en Acuicultura, Facultad de Ciencias del Mar, Universidad Católica del Norte, Coquimbo, 2010.

Person-Le Ruyet, J. Turbot culture (Scophthalmus maximus). En: Daniels, H. V. \& Watanabe, W. O. (Eds.). Practical flatfish culture and stock enhancement. Ames, Wiley-Blackwell, 2010. pp.392-412.

Puvanendran, V.; Boyce, D. \& Brown, J. Food ration requirements of 0+ yellowtail flounder Limanda ferruginea (Storer) juvenile. Aquaculture, 220(1-4):459-75, 2003.

Ricker, W. E. Growth rates and models. En: Hoar, W. S.; Randall, D. J. \& Brett, J. R. (Eds). Fish physiology. Bioenergetics and growth. Vol. 8. London, Academic Press, 1979.

Rombough, P. The effects of temperature on embryonic and larval development. In: Wood, C. M. \& McDonald, D. G. (Eds). 
PEPE-VICTORIANO, R.; SILVA, A.; VEGA, A.; ARAYA, M. \& CORNEJO, L. Efecto del aumento de la temperatura, frecuencia de alimentación y ración de alimento en el crecimiento de juveniles de turbot Psetta maxima. Int. J. Morphol., 30(3):902-907, 2012.

Society for Experimental Biology Seminar Series 61: Global Warming Implications for Freshwater and Marine Fish. Cambridge, Cambridge University Press, 1996. pp.177-223.

Ruohonen, K.; Vielma, J. \& Grove, D. J. Effects of feeding frequency on growth and food utilization of Rainbow trout [Oncorhynchus mykiss] fed low-fat herring or dry pellets. Aquaculture, 165:111-21, 1998.

Saether, B. \& Jobling, M. The effects of ration level on feed intake and growth, and compensatory growth after restricted feeding, in turbot Scophthalmus maximus L. Aquaculture Res., 30(9):647-53, 1999.

Silva, A. Culture of Chilean flounder. En: Daniels, H. V. \& Watanabe, W. O. (Eds). Practical flatfish culture and stock enhancement. Ames, Wiley-Blackwell, 2010. pp.30-45.

Sumagaysay, N. Milkfish (Chanos chanos) production and water quality in brackish water ponds at different feeding levels and frequencies. J. Appl. Ichthyol., 14(1-2):81-5, 1998.

Sokal, R. R. \& Rohlf, F. J. Biometry: Principles and practice of statistical in biological research. San Francisco, Freeman WH \& Company, 1981.p.776.

Tucker, B.; Booth, M.; Allan, G.; Booth, D. \& Fielder, S. Effects of photoperiod and feeding frequency on performance of newly weaned Australian snapper Pagrus auratus. Aquaculture, 258(1-4):514-20, 2006.

Van Ham, E. H.; Berntssen, M. H. G.; Imsland, A. K.; Parpoura, A. C.; Wendelaar Bonga, S. E. \& Stefansson, S. O. The influence of temperature and ration on growth, feed conversion, body composition and nutrient retention of juvenile turbot (Scophthalmus maximus). Aquaculture, 217(1-4):547-58, 2003.

Wang, N.; Hayward, R. \& Noltie, D. Effect of feeding frequency on food consumption, growth, size variation, and feeding pattern of age-0 hybrid sunfish. Aquaculture, 165(3-4):261-7, 1998.

\author{
Dirección para correspondencia: \\ Renzo Pepe-Victoriano \\ Centro de Recursos Naturales y Medio Ambiente \\ (CERENAYMA) \\ Departamento de Biología \\ Universidad de Tarapacá \\ Arica - CHILE
}

Email: rpepe@uta.cl

Recibido : 12-04-2012

Aceptado: 16-06-2012 